\title{
Relation between intraventricular pressure and volume in diastole
}

\author{
A L Yettram, B S Grewal, D G Gibson, J R Dawson
}

Department of Mechanical Engineering, Brunel University, Uxbridge A L Yettram B S Grewal

Cardiac Department, Brompton Hospital, London

D G Gibson

The London Hospital, London

J R Dawson

Correspondence to Professor A I Yettram, Department of Mechanical Engineering, Brunel University, Uxbridge, Middlesex UB8 3PH.

Accepted for publication 10 July 1990

\begin{abstract}
The pressure-volume curves for 10 patients with various types of heart disease were studied throughout mid to late diastole when both pressure and volume were increasing. The results were used to test a currently held theory that the form of this relation is exponential. It was found that for the patients examined this hypothesis was not valid.
\end{abstract}

It is widely believed that the left ventricular cavity becomes stiffer in diastole in disease. This reduced compliance is said to modify the filling pattern, ${ }^{1}$ raise end diastolic pressure, ${ }^{2}$ and thus limit exercise tolerance. Left ventricular compliance is measured as the rate of change of volume with pressure during the passive phase of diastole and varies throughout the filling period. ${ }^{3}$

It has been assumed for many years that the pressure-volume curve itself is exponential and that a rate constant can be calculated. ${ }^{4}$ In our experience, however, the ventricular pressure-volume curve in patients with left ventricular disease can depart considerably from any simple or uniform analytical relation. To test the hypothesis that during passive filling the left ventricular pressure-volume relation can be represented without major error by an exponential we therefore examined this relation in detail with the most satisfactory methods currently available for clinical use.

\section{Patients and methods} METHODS

We studied 10 patients, two of them on two separate occasions. The table gives their diagnoses. In all cases cardiac catheterisation was required because of standard clinical indications.

Details of patients studied

\begin{tabular}{llll}
\hline Patient & Diagnosis & Sex & Age \\
\hline 1 & Congestive cardiomyopathy & F & 60 \\
2 & Mild mitral stenosis with regurgitation & M & 27 \\
3 & Coronary artery disease & M & 60 \\
4 & Coronary artery disease with left ventricular dysfunction & M & 46 \\
5 & Coronary artery disease & M & 45 \\
6 & Coronary artery disease & M & 70 \\
7 & Coronary artery disease & M & 47 \\
$8($ a) & Coronary artery disease & M & 47 \\
$8(b)$ & Coronary artery disease with induced angina & M & 57 \\
$9($ a) & Coronary artery disease & F & 68 \\
$9(b)$ & Coronary artery disease with induced angina & & \\
10 & Aortic valve dysfunction with LV hypertrophy & &
\end{tabular}

Biplane cineangiograms were performed during held mid-inspiration $\left(60^{\circ}\right.$ left and $30^{\circ}$ right anterior oblique projections) at a frame rate of $50 / \mathrm{s}$ with $50 \mathrm{ml}$ of Urografin 370 at a flow rate of $12 \mathrm{ml} / \mathrm{s}$ injected into the ventricle. The left ventriculograms were calibrated by displacing the catheter table by $10 \mathrm{~cm}$.

Ventricular pressure was measured by a Gaeltec tip micromanometer mounted on the angiographic catheter with atmospheric pressure as zero. The micromanometer was immersed in saline for 20 minutes before use to minimise zero drift, which was found to have been less than $2 \mathrm{~mm} \mathrm{Hg}$ by the end of the study for all patients. The transducer was calibrated in situ against peak systolic and minimum diastolic pressure recorded through the angiographic lumen. Pressures were recorded photographically at a paper speed of $0 \cdot 1$ $\mathrm{m} / \mathrm{s}$ with a simultaneous electrocardiogram.

\section{ANALYSIS OF DATA}

The beats we analysed occurred within the first five after the start of contrast injection, when the haemodynamic effects of the agent itself are negligible, and were not extrasystolic or postextrasystolic. The onset of the QRS complex of the electrocardiogram was used to gate a light emitting diode and thus identify its timing on the cine film. The cineangiograms were digitised in both planes frame by frame ${ }^{5}$ throughout the cycle being studied. The timing of mitral opening was taken as the first appearance of unopacified blood through the mitral valve at the start of diastole. This was best identified on the right anterior oblique projection. Cavity volume was derived for each pair of right anterior oblique and left anterior oblique frames by computer reconstruction of the cavity. ${ }^{6}$ The two frames were aligned at the apical point, taken as the point furthest from the mid-point of the aortic root on each. The right anterior oblique view was then sliced at 50 levels perpendicular to the mid-aortic to apical line, thus providing 100 points to define the outline of the cavity. These points were then projected across to the left anterior oblique view and the intersections with its periphery were recorded. We used computer aided surface fitting techniques to reconstruct the three dimensional cavity shape and measure its enclosed volume. This method has previously been validated directly against plaster casts of left ${ }^{6}$ and right ${ }^{7}$ ventricles. Figure 1 shows some of the ventricular cavity shapes for patients in this study.

The period of passive left ventricular filling was taken as starting at the time when pres- 

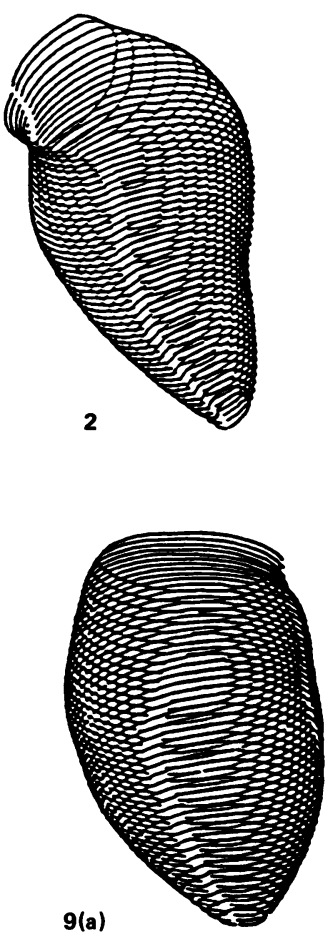
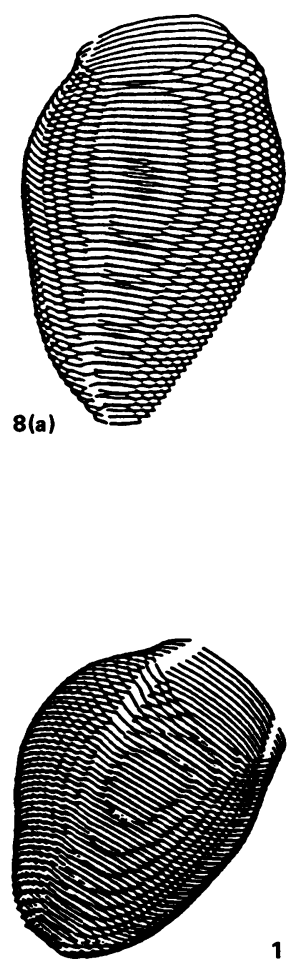

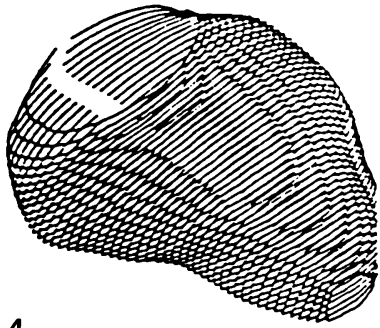

4

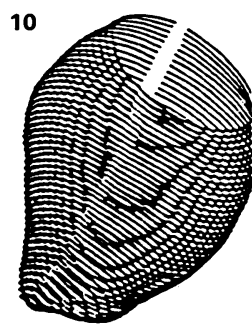

Figure 1 Typical computer aided reconstructions of the left ventricular chamber for six of the patients studied.

sure and volume started to increase simultaneously at the end of rapid filling. We chose this as a practical definition and cannot exclude the possibility of some residual tension for a short time afterwards. This contrasts with the period of rapid filling when pressure falls as volume increases. The end of passive diastole was identified as the onset of the sudden increase in filling rate associated with left atrial systole. This procedure required analysis of 5-11 frames per patient.

From this information we examined the relation between pressure and volume by regression analysis, using linear ( $P$ against $V$ ) and semilogarithmic (ln $P$ against $V$ ) plots. For each we calculated the correlation coefficient, the slope and intercepts along with their standard errors, and the standard error of the estimate. We took atmospheric pressure as zero in the semilogarithmic analysis. In line with current reported practice we did not investigate the effect of allowing the assumed zero value for pressure measurement to vary in order to improve agreement. We also examined the plot of pressure against $\mathrm{dP} / \mathrm{dV}$ (the ventricular or cavity stiffness). If the relation between pressure and volume is exponential there should be a simple linear relation between pressure and $d P / d V$, with both increasing as filling proceeds. We studied the nature of the relation in individual patients, with particular reference to its form. We noted whether $\mathrm{dP} / \mathrm{dV}$ increased uniformly throughout passive filling or whether it showed points of inflection, remaining constant or even falling as pressure rose.

\section{Results}

PRESSURE-VOLUME RELATIONS

Figure 2 shows the pressure-volume curves during passive filling for individual patients. Their shape varies greatly. Approximately half the patients showed some increase in slope towards the end of passive diastole but this was by no means uniform.

\section{REGRESSION ANALYIS}

Correlation coefficients for linear analysis of pressure against volume varied between 0.89 and 0.98 . For semilogarithmic analysis they varied between 0.83 and 0.99 . There was no significant difference between values derived from the two approaches when individual values were compared. The standard error of estimated volume derived from pressure meaurements was $3.7(1.9) \mathrm{mm} \mathrm{Hg}$ when logarithmic analysis was used and 4.0 (1.6) $\mathrm{mm} \mathrm{Hg}$ with linear analysis. Again there was no significant difference between these values.

\section{PRESSURE-CAVITY STIFFNESS RELATION}

Figure 3 shows curves from individual patients. A progressive increase in cavity stiffness (not necessarily linear) with pressure was seen in only four. In the remainder the curve was sigmoid with stiffness falling rather than rising for at least part of the period of pressure rise. 
Figure 2 Portions of pressure-volume curves for all 12 studies. Pressure and volume were both rising in diastole.
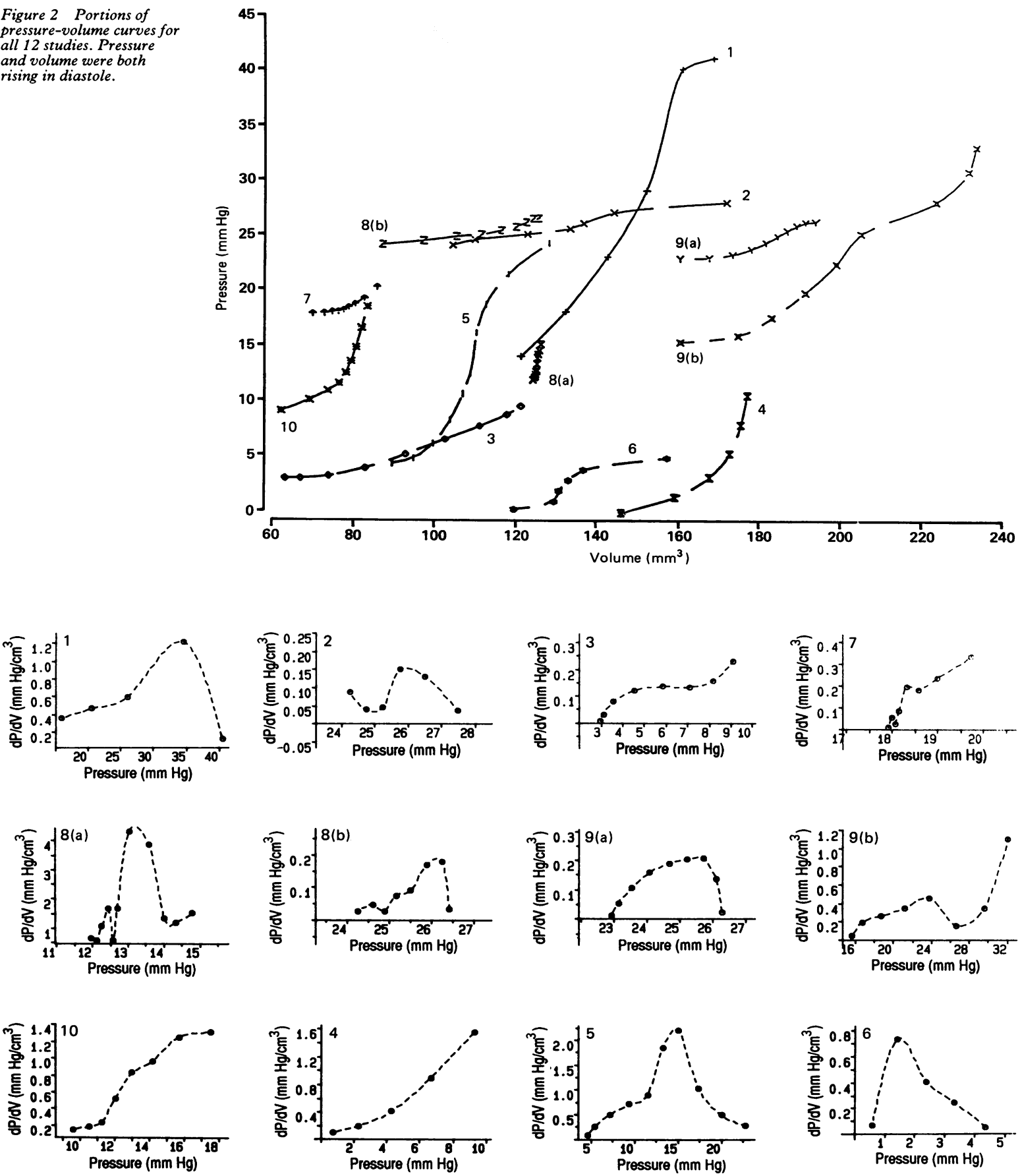

Figure 3 Plots of $d P / d V$ against $P$ for each patient.

Discussion

The stiffness of the left ventricular cavity during the passive phase of diastole is estimated from its pressure-volume relations. The curve of pressure against volume is often not linear, with the slope increasing towards end diastole. It has therefore become traditional, since the original studies of Noble et $a l,{ }^{4}$ to use an exponential analysis and plot the logarithm of pressure against volume. This approach has affected the way in which diastolic function is understood. Unlike other mathematical functions exponential analysis allows a rate constant to be calculated; in the present context it is usually referred to as a stiffness constant and used by some to express 
ventricular stiffness itself. Departures of the curve from the assumed exporential have been taken as evidence that some additional mechanism is influencing pressure-volume relations; the presence and even the magnitudes of viscous forces have been calculated on this basis by Eichhorn et al. ${ }^{8}$

We undertook the present study because we were impressed by how often the pressurevolume relations in patients with left ventricular disease departed from any simple or uniform relation. We deliberately excluded the rapid filling period and also atrial systole from consideration, when other mechanisms might have been acting and when viscous effects could be invoked. The methods we used were the most satisfactory available clinically and our approach to volume estimation, in particular, was not subject to limitations imposed by single plane angiography or assumed geometry. In common with virtually all other studies, we measured ventricular pressure at only one site within the ventricle, a questionable procedure if intraventricular pressure differences develop within the cavity. Such differences would be expected at times of rapid filling and indeed have been documented. During the passive phase of diastole, however, the filling rate is slow, and there is no theoretical or experimental reason to believe that they are present. Our results clearly showed that the relation between pressure and cavity stiffness departed very significantly from the assumed exponential. Even the undemanding criterion that ventricular stiffness should increase, not necessarily linearly, with pressure was met in only one third of the patients. In eight, calculated cavity stiffness actually fell with increasing pressure over part of the period being examined. We conclude that critical examination in patients with left ventricular disease showed that this exponential analysis was of very limited value.

Influences external to the left ventricle may have distorted our results, thus causing the underlying exponential to be obscured. It has been suggested that measured cavity stiffness might be modified by pericardial restraint. ${ }^{9}$ While this undoubtedly occurs in a few patients after open heart surgery, ${ }^{10}$ we do not feel that it was important in our patients. Pericardial restraint is an end diastolic phenomenon, occurring only late in ventricular filling. It would therefore appear as a sudden increase in calculated stiffness. We found no evidence of this: indeed in six of our patients stiffness was actually falling in the cine frames before the onset of atrial systole. In addition, all our patients showed a further volume increase with atrial contraction that would be out of the question if the ventricle were restrained by a structure as unyielding as the pericardium. Right ventricular disease has also been invoked as a possible mechanism influencing left ventricular pressure-volume relations. Again, we consider this to have been unlikely in our patients most of whom had dominantly left ventricular disease. The influence of the right ventricle is mediated largely through the pericardium ${ }^{11}$; any more direct action is associated with distortion of the left ventricle which was not present. We may have been incorrect to take atmospheric as the zero for pressure measurement, though this has been assumed in all previous studies. During isovolumic relaxation, however, the observed time course of the declining pressure can be made to agree more closely with an assumed exponential if the asymptote as well as the time constant is allowed to vary. ${ }^{12}$ In normal subjects the effective zero for pressure measurement during this period has been found to be large and negative, often as much as $50-60 \mathrm{~mm} \mathrm{Hg}$ below atmospheric. We do not think that manipulating asymptotes in this way would have improved agreement during the passive phase of diastole. The only way in which it could have done so in the patients in whom stiffness was declining towards end diastole was for the effective zero to have actually risen above the value actually measured in the ventricle, a suggestion not worthy of being considered further.

It does, however, bring to light a possible paradox. How can the large negative asymptote during isovolumic relaxation suggested by exponential analysis be reconciled with the assumed atmospheric one during filling? Is one or other incorrect or has there been a change? If the latter is true, what is the time course of this change and how does it affect predictions about "incomplete relaxation" that depend on extrapolation of the exponential curve derived during isovolumic relaxation into the period of filling ? $^{13}$ Clearly there are major inconsistencies between the two sets of results that in our opinion merely underline the unsatisfactory nature of both.

Our results emphasise the complex nature of diastolic left ventricular pressure-volume relations. Even if the isolated left ventricular pressure-volume curve is exponential when heart function is normal there is every reason to suppose that it departs from this in the intact patient with disease. Ventricular involvement is frequently non-uniform, particularly in coronary artery disease, whereas hypertrophy leads to major structural abnormalities. Even in the normal heart the structure of the myocardium is very highly organised with fibre angle varying across the wall and in different regions of the ventricle. Changes in transverse dimension are mediated more by myocardial thickening or thinning than by changes in fibre length. ${ }^{14}$

We are dealing with a thick-walled pressure vessel with a complex wall structure that is highly orthotropic in nature. The rate of volume change with pressure will depend not only on the geometry but also on the stiffness of the material of the myocardium. This material stiffness itself may not be constant but could well vary with time and thus with volume, possibly in some non-linear way. With this in mind it is not surprising that the pressurevolume relation in diastole does not follow a simple exponential law. Relaxation may even persist, to some degree, beyond the period of rapid filling into the period that we have considered. Indeed we found that myocardial 
stiffness did vary during passive filling and our findings in this respect will be described in a future paper. Thus the diversity of passive diastolic relations seen in a small number of patients with heart disease is not surprising. We suggest that this diversity be accepted and if possible analysed rather than effectively suppressed by procrustean assumptions. Exponential analysis has not contributed significantly to an understanding of left ventricular diastolic behaviour and we suggest that it be abandoned.

1 Danford DA, Huhta JC, Murphy DJ. Doppler echocardiographic approaches to ventricular diastolic function. Echocardiogr 1986;3:33-40.

2 Braunwald E, Ross Jr J. The ventricular end-diastolic pressure: an appraisal of its value in the recognition of pressure: an appraisal of its value in the recognition
ventricular failure in man. Am J Med 1983;34:147.

3 Mirsky I. Assessment of passive elastic stiffness of cardiac muscle: mathematical concepts, physiologic and clinical considerations, direction of future research. Prog Car diovasc Dis 1976;18:277-308.

4 Noble MIM, Milne ENC, Goerke RJ, et al. Left ventricular filling and diastolic pressure-volume relations in the conscious dog. Circ Res 1969;24:269-83.
5 Gibson DG, Prewitt TA, Brown DJ. Analysis of left ventricular wall movement during isovolumic relaxation and its relation to coronary artery disease. Br Heart $J$ 1976;38:1010-9.

6 Yettram AL, Vinson CA, Gibson DG. Computer modelling of the human left ventricle. Trans $A S M E$ 1982;104: 148-52.

7 Redington AN, Gray HH, Hodson ME, Rigby ML, Oldershaw PJ. Characterisation of the normal right ventricular pressure-volume relation by biplane angiography and simultaneous micromanometer pressure measurements. Br Heart J 1988;59:23-30

8 Eichhorn P, Grimm J, Koch R, Hess O, Carroll J, Krayenbuehl HP. Left ventricular relaxation in patients with left ventricular hypertrophy secondary to aortic valve disease. ventricular hypertrophy secon
Circulation 1982;65:1395-404.

9 Smiseth OA, Frais MA, Kingma I, Smith ER, Tyberg JV. Assessment of pericardial constraint in dogs. Circulation 1985;71:158-64

10 St John Sutton MG, Gibson DG. Measurement of postoperative pericardial pressure in man $\mathrm{Br}$ Heart $J$ 1977;39:1-6.

11 Ludbrook PA, Byrne JD, McKnight RC. Influence of right ventricular hemodynamics on left ventricular diastolic pressure-volume relations in man. Circulation 1979; 59:21-31.

12 Thompson DS, Waldrom CB, Coltart DJ, Jenkins BS, Webb-Peploe MM. Estimation of time constant of left Webb-Peploe MM. Estimation of time constant of left

13 Weisfeldt ML, Weiss JL, Frederiksen JT, Yin FCP. Quantification of incomplete left ventricular relaxation: relationship to the time constant for isovolumic pressure fall. Eur Heart J 1980;1:A119-29.

14 Sallin EA. Fiber orientation and ejection fraction in the human left ventricle. Biophys $J$ 1969;9:954-64. 